\title{
Single cut integration
}

\section{Ruth Britto and Edoardo Mirabella}

Institut de Physique Théorique, CEA-Saclay, Orme des Merisiers, F-91191, Gif-sur-Yvette cedex, France

E-mail: ruth.britto@cea.fr, edoardo.mirabella@cea.fr

ABstract: We present an analytic technique for evaluating single cuts for one-loop integrands, where exactly one propagator is taken to be on shell. Our method extends the double-cut integration formalism of one-loop amplitudes to the single-cut case. We argue that single cuts give meaningful information about amplitudes when taken at the integrand level. We discuss applications to the computation of tadpole coefficients.

KEYWORDS: NLO Computations, QCD 


\section{Contents}

1 Introduction $\quad 1$

2 Evaluation of the single cut $\quad 2$

$\begin{array}{lll}3 & \text { Single cut of general integrands } & 7\end{array}$

4 Computation of tadpole coefficients $\quad 9$

5 Massless external legs $\quad 15$

$\begin{array}{llr}6 & \text { Conclusions and discussion } & 19\end{array}$

$\begin{array}{ll}\text { A Phase space parametrization } & 20\end{array}$

\section{Introduction}

Precision calculations in gauge theories such as QCD are needed for observations in hadron collider experiments and are an important motivation for studies of higher-order scattering in general. Recently there have been rapid developments in computational techniques, many centered around so-called unitarity methods [1, 2].

The idea underlying the unitarity methods is to constrain amplitudes by their branch cuts in various channels. The constraints from cuts are sufficient only for certain classes of amplitudes, such as one-loop amplitudes in massless theories [3]. Unitarity cuts are evaluated by the Cutkosky rules [4], which put two propagators on shell. Rather than completing the dispersion integral at this point, unitarity methods store the imaginary part of the amplitude obtained this way, and accumulate similar information from all possible cuts in analytic continuations to other kinematic regions.

Putting more than two propagators of a one-loop amplitude on shell gives a "generalized" unitarity cut [5-16]. It isolates the part of the amplitude from diagrams containing the specific propagators being cut. An advantage of such cuts is that the delta functions that are used to place the propagators on shell effectively reduce the dimension of the remaining integral. So these generalized cuts are relatively easy to evaluate but give information about a correspondingly smaller part of the full amplitude. However, generalized unitarity can also be taken in the other direction, by cutting just one propagator. This is the "single cut" we discuss in this paper. Our focus differs from previous studies [17-20] in that we are interested in the explicit evaluation of the 4-dimensional single-cut integral, with standard Feynman propagators, with a view towards computing tadpole coefficients in amplitudes with internal masses. 
One-loop amplitudes have an expansion in terms of master integrals, which are scalar box, triangle, bubble and tadpole integrals, with coefficients that are rational functions of the kinematic invariants [21-36]. In practice, unitarity methods operate by pattern matching cuts of amplitudes with cuts of master integrals. Tadpole integrals lack cuts in physical channels (double cuts), but we hope that single cuts should be useful to calculate their coefficients. In principle, single cuts should give information about all parts of the amplitude. We study the single cuts of master integrals and find that the tadpole gives a rational value, while the others have purely logarithmic single cuts. Therefore the tadpole coefficients can be targeted by discarding all logarithms. Other approaches to analytic computation of tadpole coefficients have been proposed, using universal UV and IR divergent behavior [37, 38] or introducing an auxiliary propagator [39].

Inspired by the formalism for explicit evaluation of double cuts [40-48], where the two-dimensional (or $(D-2)$-dimensional) integral is performed algebraically by the Cauchy residue theorem, we rewrite our loop momentum in terms of spinor variables, which can in turn be exchanged for a complex variable and a real parameter. Since we now have fewer cut constraints than in a double cut, we are not able to evaluate the integral over the real parameter trivially. In fact, we leave it unevaluated. We find that it is sufficient to work at the integrand level; moreover, the full single cut integral will typically diverge. (Supersymmetric theories are a notable exception: there, single cuts are well defined [18] and can be used to check expressions for planar multi-loop amplitudes [49].) The integral over the complex variable is addressed by the Generalized Cauchy Theorem, invoked similarly in [48]. If we are computing single cuts to find tadpole coefficients, we see another major difference compared to double cuts. While double cut evaluations were a matter of evaluating residues at poles, here it is the contour integral part of the formula that dominates the tadpole contribution, so we do not compute residues at all.

Working at the integrand level is important because functions of the loop momentum can have non-vanishing single cuts even if they integrate to zero [18]. Therefore, a proper expansion of the integrand from a Feynman diagram includes all such terms, which have been thoroughly classified in the context of four-dimensional reduction [10, 50].

The paper is organized as follows. In section 2, we explain the phase space integration for single cuts and evaluate the cuts for master integrals. In section 3 , we present the single cuts of the first few types of integrands with tensor numerators. In section 4, we extract the tadpole coefficients in some sample tensor integrands. In section 5, we discuss the modifications necessary in the case of massless external legs (which are vanishing Gram determinants for bubble integrals). The appendix contains further details on the phase space parametrization.

\section{Evaluation of the single cut}

Our starting point is the one-loop integrand,

$$
I=\frac{N(k)}{D_{0} D_{1} \cdots D_{k}}
$$


where $N(k)$ is a polynomial in the loop momentum $k$, and the denominator factors are

$$
D_{i}=\left(k-K_{i}\right)^{2}-m_{i}^{2} .
$$

The single cut is a singularity of the amplitude selecting single propagators. We define the 4-dimensional single-cut operator for a particular propagator $D_{i}$ to act on the integrand as

$$
\Delta_{D_{i}}[I] \equiv \int d^{4} k \delta^{(+)}\left(D_{i}\right)\left(\frac{N(k)}{D_{0} \cdots D_{i-1} D_{i+1} \cdots D_{k}}\right) .
$$

The single cut must be applied to the integrand, because there are non-vanishing contributions from so-called spurious terms (terms that vanish upon integration). Working with the integrand allows us to identify the particular propagator being cut.

To evaluate the single cut analytically, we introduce convenient variables and reference vectors following $[42,48,51]$. First, we would like to exchange the original loop momentum variable $k$ for a null vector $\ell_{1}$ in order to make use of the spinor formalism [52-56]. With respect to an arbitrary Lorentz vector $K$ satisfying $K^{2} \neq 0$ and $K_{0}>0$, we decompose the loop momentum variable,

$$
k=\ell_{1}+\xi K,
$$

where $\xi$ is a scalar factor taking a value such that $\ell_{1}$ is null. This condition is implemented by another delta function and an integral over $\xi$,

$$
\int d^{4} k(\bullet)=\int d \xi \int d^{4} \ell_{1} \delta^{(+)}\left(\ell_{1}^{2}\right)\left(2 \ell_{1} \cdot K\right)(\bullet)
$$

Let us now look at the single cut integral, (2.3). For simplicity, suppose we have redefined the loop momentum so that $K_{i}=0$. We comment further on allowable redefinitions below. Also, let $m=m_{i}$. Applying the change of variables (2.4), we have

$$
\int d^{4} k \delta^{(+)}\left(k^{2}-m^{2}\right)(\bullet)=\int d \xi \int d^{4} \ell_{1} \delta^{(+)}\left(\ell_{1}^{2}\right) \frac{2 \ell_{1} \cdot K}{\sqrt{\Delta}} \delta(\xi-\bar{\xi})(\bullet),
$$

where

$$
\Delta=\left(2 \ell_{1} \cdot K\right)^{2}+4 K^{2} m^{2}, \quad \bar{\xi}=\frac{-2 \ell_{1} \cdot K+\sqrt{\Delta}}{2 K^{2}} .
$$

We continue by exchanging the integration of $\ell_{1}$ over the lightcone for an integral over the complex plane and a real parameter $t$. First, we express the vector $K$ as a sum of two null vectors, $p$ and $q$, which will later allow us to integrate over familiar complex variables instead of spinors. Since our choice of $K$ was arbitrary, we can consider the choice of null $p$ and $q$ to be the starting point, making sure that $p \cdot q \neq 0$ and $p_{0}+q_{0}>0$.

$$
K=p+q, \quad p^{2}=q^{2}=0 .
$$

The replacement for the loop momentum is

$$
\ell_{1}^{\mu}=t\left(p^{\mu}+z \bar{z} q^{\mu}+\frac{z}{2}\left\langle q\left|\gamma^{\mu}\right| p\right]-\frac{\bar{z}}{2}\left\langle p\left|\gamma^{\mu}\right| q\right]\right) .
$$


Since $2 p \cdot q=K^{2}$ and $2 \ell_{1} \cdot K=t(1+z \bar{z}) K^{2}$, the integral measure becomes

$$
\int d^{4} k \delta^{(+)}\left(k^{2}-m^{2}\right)(\bullet)=\int d \xi \int_{0}^{\infty} \frac{d t}{4} \int(i d z \wedge d \bar{z}) \frac{K^{2} t^{2}(1+z \bar{z}) \delta(\xi-\bar{\xi})}{\sqrt{t^{2}(1+z \bar{z})^{2}+u}}(\bullet),
$$

where now

$$
\bar{\xi}=\frac{u}{2} \frac{1}{\sqrt{t^{2}(1+z \bar{z})^{2}+u}+t(1+z \bar{z})}
$$

and we have defined

$$
u \equiv \frac{4 m^{2}}{K^{2}}
$$

We will find it convenient to work in the limit $u \rightarrow 0$, equivalent to choosing our arbitrary $K$ such that $K^{2} \gg m^{2}$. In fact, we will be able to set $u=0$ exactly in all the cuts we study.

Now, the $\xi$ integral can be performed immediately by the delta function substitution. The complex integration over $z$ and $\bar{z}$ will be performed by the Generalized Cauchy Formula as described in [48]. That is, for the integrand $F(z, \bar{z})$ we construct a primitive $G(z, \bar{z})$ with respect to, say, $\bar{z}$. Let $D$ be a disk in the complex plane encompassing all poles of $G(z, \bar{z})$ viewed as a function of $z$. Then

$$
\int_{D} F(z, \bar{z}) d \bar{z} \wedge d z=\oint_{\partial D} d z G(z, \bar{z})-2 \pi i \sum_{\text {poles } z_{j}} \operatorname{Res}\left\{G(z, \bar{z}), z_{j}\right\}
$$

In practice, we use $\Lambda$ to denote the radius of the disk $D$ and rewrite the complex variable in terms of polar coordinates:

$$
z=r e^{i \alpha} ; \quad D=\{(r, \alpha) \mid 0 \leq r \leq \Lambda, 0 \leq \alpha<2 \pi\}
$$

Additional details on the phase space parametrization are given in the appendix.

The final integration over $t$ will not actually be carried out; we extract the information we need at the integrand level. In fact, the integral over $t$ typically diverges. It should be cut off both from above and below, and any further transformations should be consistent with such a cutoff. For example, we allow linear shifts of the original loop momentum, but not global rescalings.

Analytically, the $t$-dependence of the cut integrands turns out to be useful, as we can restrict our attention to leading or subleading terms in $t$ in order to compute tadpole coefficients.

In our first change of variables for the single cut, (2.5), we assumed that the cut propagator was already in the form $\left(k^{2}-m^{2}\right)$. In fact, it is good to redefine the loop momentum $k \rightarrow k+K_{i}$ in each term of the single cut operation (2.3) so that the delta function is $\delta^{(+)}\left(k^{2}-m^{2}\right)$. Because the single cut integral diverges, this redefinition is most obviously valid in the limit of large $K^{2}$, which we will implement routinely. Otherwise, we must pay attention to the details of the cutoffs.

We will now study the single cuts of the integrands of master integrals. 


\section{Single cut of tadpole}

The scalar tadpole is the simplest integrand allowing the single cut operation. With the variables of $(2.7)$,

$$
\Delta_{D_{0}}\left[\frac{1}{k^{2}-m_{0}^{2}}\right]=\int \frac{d t}{4} \int(i d z \wedge d \bar{z}) \frac{K^{2} t^{2}(1+z \bar{z})}{\sqrt{t^{2}(1+z \bar{z})^{2}+u}} .
$$

One $\bar{z}$-primitive of the integrand is

$$
\mathcal{P}_{1} \equiv(1 / z) K^{2} \sqrt{t^{2}(1+z \bar{z})^{2}+u},
$$

so the result of applying the Generalized Cauchy Formula (2.9) is

$$
(2 \pi) K^{2}\left(\sqrt{t^{2}\left(1+\Lambda^{2}\right)^{2}+u}-\sqrt{t^{2}+u}\right) .
$$

The factor of $i$ has dropped out in converting the differential form (see the appendix for details).

In the limit of vanishing $u$, the single cut of the tadpole is therefore

$$
(2 \pi) K^{2} t\left(\Lambda^{2}+\frac{u}{2 t^{2}\left(1+\Lambda^{2}\right)}-\frac{u}{2 t^{2}}\right) .
$$

We omit writing the integral over $t$ (and the factor of 4 in the denominator), since it will not be necessary in this paper to carry out this integration. In subsequent integrals we will also drop all subleading terms in $u$ so that the formulas are more manageable, while still sufficiently distinct.

\section{Single cut of bubble}

Consider the integrand $1 /\left(D_{0} D_{1}\right)$, and take the single cut of $D_{0}$.

$$
\Delta_{D_{0}}\left[\frac{1}{\left(k^{2}-m_{0}^{2}\right)\left(\left(k-K_{1}\right)^{2}-m_{1}^{2}\right)}\right]=\int d^{4} k \delta^{(+)}\left(k^{2}-m_{0}^{2}\right) \frac{1}{D_{1}} .
$$

Now, under the integral with the delta function,

$$
D_{1}=f_{1}-2 \ell_{1} \cdot K_{1}-2 \bar{\xi} K \cdot K_{1},
$$

where

$$
f_{i} \equiv K_{i}^{2}-m_{i}^{2}+m_{0}^{2}
$$

In the limit of vanishing $u$, we also have $\bar{\xi} \rightarrow 0$, so we can neglect the last term of (2.11). ${ }^{1}$ So we proceed with the replacement $D_{1}=f_{1}-t\left\langle\lambda\left|K_{1}\right| \tilde{\lambda}\right]$, followed by expansion in the null vectors $p$ and $q$. The single cut is

$$
\int(i d z \wedge d \bar{z}) \frac{K^{2} t}{\mathcal{F}\left(K_{1}, z, \bar{z}\right)}
$$

\footnotetext{
${ }^{1}$ Indeed, one can check using (2.8) that in the rest frame $K=\left(K_{0}, 0,0,0\right)$, this term falls off as $1 / K_{0}$.
} 
where we have defined

$$
\mathcal{F}\left(K_{i}, z, \bar{z}\right) \equiv f_{i}-t\left(\left\langle p\left|K_{i}\right| p\right]+\bar{z}\left\langle p\left|K_{i}\right| q\right]+z\left\langle q\left|K_{i}\right| p\right]+z \bar{z}\left\langle q\left|K_{i}\right| q\right]\right) .
$$

One $\bar{z}$-primitive of the integrand in (2.13) is given by

$$
\mathcal{P}_{2} \equiv-\frac{K^{2} \log \mathcal{F}\left(K_{1}, z, \bar{z}\right)}{\left\langle p\left|K_{1}\right| q\right]+z\left\langle q\left|K_{1}\right| q\right]} .
$$

\section{Single cut of triangle and box}

The analysis of triangle and box integrands is similar to the bubble. For the triangle integrand, $1 /\left(D_{0} D_{1} D_{2}\right)$, the single cut can be expressed in terms of the primitive, given by

$$
\begin{aligned}
\mathcal{P}_{3} & =\int d \bar{z} \frac{K^{2} t}{\mathcal{F}\left(K_{1}, z, \bar{z}\right) \mathcal{F}\left(K_{2}, z, \bar{z}\right)} \\
& =-\frac{K^{2}}{\mathcal{D}\left(K_{1}, K_{2}, z\right)} \log \left(\frac{\mathcal{F}\left(K_{1}, z, \bar{z}\right)}{\mathcal{F}\left(K_{2}, z, \bar{z}\right)}\right) .
\end{aligned}
$$

For the box $1 /\left(D_{0} D_{1} D_{2} D_{3}\right)$, the $\bar{z}$-primitive is given by

$$
\begin{aligned}
\mathcal{P}_{4}= & \int d \bar{z} \frac{K^{2} t}{\mathcal{F}\left(K_{1}, z, \bar{z}\right) \mathcal{F}\left(K_{2}, z, \bar{z}\right) \mathcal{F}\left(K_{3}, z, \bar{z}\right)} \\
= & \frac{K^{2}\left\langle\lambda\left|K_{1}\right| q\right] \log \mathcal{F}\left(K_{1}, z, \bar{z}\right)}{\mathcal{D}\left(K_{1}, K_{2}, z\right) \mathcal{D}\left(K_{3}, K_{1}, z\right)}+\frac{K^{2}\left\langle\lambda\left|K_{2}\right| q\right] \log \mathcal{F}\left(K_{2}, z, \bar{z}\right)}{\mathcal{D}\left(K_{2}, K_{3}, z\right) \mathcal{D}\left(K_{1}, K_{2}, z\right)} \\
& +\frac{K^{2}\left\langle\lambda\left|K_{3}\right| q\right] \log \mathcal{F}\left(K_{3}, z, \bar{z}\right)}{\mathcal{D}\left(K_{3}, K_{1}, z\right) \mathcal{D}\left(K_{2}, K_{3}, z\right)}
\end{aligned}
$$

We have defined

$$
\mathcal{D}\left(K_{i}, K_{j}, z\right) \equiv f_{j}\left\langle\lambda\left|K_{i}\right| q\right]-f_{i}\left\langle\lambda\left|K_{j}\right| q\right]-t[p q]\left\langle\lambda\left|K_{i} K_{j}\right| \lambda\right\rangle, \quad \text { where }|\lambda\rangle=|p\rangle+z|q\rangle .
$$

\section{Overview: master integrands and strategy for single cuts}

We have found the primitives $\mathcal{P}_{1}, \mathcal{P}_{2}, \mathcal{P}_{3}, \mathcal{P}_{4}$, which are associated to the various master integrands. The single-cut calculation would be completed by the Generalized Cauchy formula, (2.9). It turns out that in the limit $\Lambda \rightarrow \infty$, we can ignore the residues and restrict our attention to the closed line integral term. In polar coordinates,

$$
\oint_{\partial D} \mathcal{P}_{n}(z, \bar{z}) d z=i \int_{0}^{2 \pi} d \alpha \Lambda e^{i \alpha} \mathcal{P}_{n}\left(\Lambda e^{i \alpha}, \Lambda e^{-i \alpha}\right)
$$

In the limit $\Lambda \rightarrow \infty$, the leading behavior of the integrands is

$$
\begin{aligned}
\Lambda e^{i \alpha} \mathcal{P}_{1} & \simeq \Lambda^{2} t \\
\Lambda e^{i \alpha} \mathcal{P}_{n} & \simeq \frac{\log \left(\Lambda^{2}\right)}{\Lambda^{n-2}}, \quad n=2,3,4 .
\end{aligned}
$$

The higher-point integrands are suppressed by powers of $\Lambda$. Moreover, among all these primitives, all rational terms come from tadpoles. Tadpole primitives are purely rational, 
while the others are purely logarithmic. Therefore, in an algorithm targeting tadpole coefficients, we will select terms of the single cut with specific dependence on $\Lambda^{2}$.

For fully generic integrands, we only need the single-cut operator selecting the terms proportional to $\Lambda^{2} t$, which we denote by a bar.

$$
\left.\bar{\Delta}_{D_{i}}[I] \equiv \Delta_{D_{i}}[I]\right|_{\Lambda^{2} t-\text { terms }} .
$$

For integrands with null external momenta, it is convenient to define some further refinements of the operator, as follows.

$$
\begin{aligned}
& \left.\tilde{\Delta}_{D_{0}}[I] \equiv \Delta_{D_{0}}[I]\right|_{\Lambda^{4} t^{2}-\text { terms }} . \\
& \left.\hat{\Delta}_{D_{0}}[I] \equiv \Delta_{D_{0}}[I]\right|_{\log \left(\left\langle q\left|K_{1}\right| q\right] \Lambda^{2}\right) \text {-terms }}
\end{aligned}
$$

In the case where the masses of the propagators are not all distinct, we may also want to collect the single cuts of all propagators with a given mass, as indicated by the subscript $m^{2}$.

$$
\bar{\Delta}_{m^{2}}[I] \equiv \sum_{i: m_{i}=m} \bar{\Delta}_{D_{i}}[I]
$$

It is now clear how to distinguish single cuts of the various master integrands. Since spurious terms give nonvanishing single cuts as well, we need to know them in more detail. We now compute the single cuts of some integrands in general form. The results will allow us to compute single cuts of spurious terms on one hand and the single cut of a total integrand expansion on the other.

\section{Single cut of general integrands}

In listing the single-cut results of general integrands, it is as convenient and more useful to list them in terms of general numerators and denominators. A general integrand will take the form

$$
I_{n, p}\left(A_{1}, \ldots, A_{p} ; D_{0}, \ldots, D_{n-1}\right) \equiv \frac{\prod_{i=1}^{p}\left(2 k \cdot A_{i}\right)}{\prod_{j=0}^{n-1} D_{j}},
$$

since any appearance of the contraction $k^{2}$ in the numerator can be replaced by $m_{0}^{2}$ in the single cut. Using (2.7) in the limit $u \rightarrow 0$, we see that the single cut of $D_{0}$ gives

$$
\Delta_{D_{0}}\left[I_{n, p}\right]=K^{2} t^{p+1} \int_{0}^{2 \pi} d \alpha G_{n, p}\left(A_{1}, \ldots, A_{p} ; D_{0}, \ldots, D_{n} ; \alpha\right),
$$

where we have defined

$$
G_{n, p}\left(A_{1}, \ldots, A_{p} ; D_{0}, \ldots, D_{n-1} ; \alpha\right) \equiv \Lambda e^{i \alpha}\left[\int d \bar{z} \frac{\prod_{i=1}^{p}\left\langle\lambda\left|A_{i}\right| \tilde{\lambda}\right]}{\prod_{j=1}^{n-1}\left(f_{j}-t\left\langle\lambda\left|K_{j}\right| \tilde{\lambda}\right]\right)}\right]_{z \rightarrow \Lambda e^{i \alpha}, \bar{z} \rightarrow \Lambda e^{-i \alpha}} .
$$

The spinors $\langle\lambda|$ and $\mid \tilde{\lambda}]$ depend on $z$ and $\bar{z}$ as follows,

$$
\langle\lambda|=\langle p|+z\langle q|, \quad| \tilde{\lambda}]=| p]+\bar{z}| q] .
$$

The single cut of $I_{n, p}$ is known once the integral $G_{n, p}$ has been computed. We now compute the single cut of $I_{n, p}$ for several values of $(n, p)$. 


\section{Computation of $\bar{\Delta}_{\mathrm{D}_{0}}\left[\mathrm{I}_{1,1}\right]$}

It is easy to show that

$$
G_{1,1}\left(A_{1} ; D_{0} ; \alpha\right)=\Lambda^{2}\left\langle p\left|A_{1}\right| p\right]+\Lambda^{3} e^{i \alpha}\left\langle q\left|A_{1}\right| p\right]+\frac{\Lambda^{3}}{2} e^{-i \alpha}\left\langle p\left|A_{1}\right| q\right]+\frac{\Lambda^{4}}{2}\left\langle q\left|A_{1}\right| q\right] .
$$

Therefore, paying attention to the powers of $t$ included in (3.2), we find the following values for the first two refinements of single-cut operators.

$$
\begin{aligned}
& \bar{\Delta}_{D_{0}}\left[I_{1,1}\left(A_{1} ; D_{0}\right)\right]=0 . \\
& \tilde{\Delta}_{D_{0}}\left[I_{1,1}\left(A_{1} ; D_{0}\right)\right]=\pi K^{2} \Lambda^{4}\left(2 A_{1} \cdot q\right) t^{2} .
\end{aligned}
$$

\section{Computation of $\bar{\Delta}_{\mathrm{D}_{0}}\left[\mathbf{I}_{2,1}\right]$}

The $\Lambda^{2} t$-part of the single cut (3.2) is the $\Lambda^{2} / t$ part of $G_{2,1}$, which is

$$
-\frac{\Lambda^{2}}{t} \frac{A_{1} \cdot q}{K_{1} \cdot q}
$$

The single cut of $I_{2,1}$ is obtained after the trivial integration over $\alpha$, giving

$$
\bar{\Delta}_{D_{0}}\left[I_{2,1}\left(A_{1} ; D_{0}, D_{1}\right)\right]=-\frac{A_{1} \cdot q}{K_{1} \cdot q} \Delta_{D_{0}}\left[\frac{1}{D_{0}}\right] .
$$

In the $u \rightarrow 0$ limit, $\bar{\Delta}_{D_{0}}\left[I_{2,1}\left(A_{1} ; D_{0}, D_{1}\right)\right]$ is therefore proportional to the single cut of the tadpole.

For later convenience we compute $\hat{\Delta}_{D_{0}}\left[I_{2,1}\right]$ and $\bar{\Delta}_{m^{2}}\left[I_{2,1}\right]$ in the case where $K_{1}^{2}=0$ and $m_{0}^{2}=m_{1}^{2}=m^{2}$. The logarithmic part of $G_{2,1}$ is a lengthy expression. The logarithmic part of of the single cut of $I_{2,1}$ is

$$
\begin{aligned}
\hat{\Delta}_{D_{0}}\left[I_{2,1}\left(A_{1} ; D_{0}, D_{1}\right)\right]= & 2 \pi K^{2} t\left[\frac{\left\langle p\left|K_{1}\right| q\right]\left\langle q\left|A_{1}\right| p\right]}{\left\langle q\left|K_{1}\right| q\right]^{2}}+\frac{\left\langle p\left|K_{1}\right| p\right]\left\langle q\left|A_{1}\right| q\right]}{\left\langle q\left|K_{1}\right| q\right]^{2}}\right. \\
& \left.+\frac{\left\langle p\left|A_{1}\right| q\right]\left\langle q\left|K_{1}\right| p\right]}{\left\langle q\left|K_{1}\right| q\right]^{2}}-\frac{2\left\langle p\left|K_{1}\right| q\right]\left\langle q\left|A_{1}\right| q\right]\left\langle q\left|K_{1}\right| p\right]}{\left\langle q\left|K_{1}\right| q\right]^{3}}-\frac{\left\langle p\left|A_{1}\right| p\right]}{\left\langle q\left|K_{1}\right| q\right]}\right] \\
\equiv & \sum_{a} c_{a}(t) v_{a}^{\mu} A_{1 \mu} .
\end{aligned}
$$

The last line is simply an abbreviation for the expression, which will be convenient shorthand in one of the examples we give in section 5. In that case where $K_{1}^{2}=0$ and $m_{0}^{2}=m_{1}^{2}=m^{2}$, we also have

$$
\bar{\Delta}_{m^{2}}\left[I_{2,1}\left(A_{1} ; D_{0}, D_{1}\right)\right]=\bar{\Delta}_{D_{0}}\left[I_{2,1}\left(A_{1} ; D_{0}, D_{1}\right)\right]+\bar{\Delta}_{D_{1}}\left[I_{2,1}\left(A_{1} ; D_{0}, D_{1}\right)\right]=0 .
$$

The single cut $\bar{\Delta}_{D_{1}}$ is computed redefining the loop momentum $k \rightarrow k+K_{1}$, as described in section 2 . 


\section{Computation of $\bar{\Delta}_{\mathrm{D}_{0}}\left[\mathbf{I}_{2,2}\right]$}

The expression of $G_{2,2}$ is rather complicated and it is not shown here. We obtain $\bar{\Delta}_{D_{0}}\left[I_{2,2}\right]$ (or $\tilde{\Delta}_{D_{0}}\left[I_{2,2}\right]$ ) by expanding $K^{2} t^{3} G_{2,2}$ in the limit $\Lambda \rightarrow \infty$ and then integrating the $\Lambda^{2} t$-terms (or $\Lambda^{4} t^{2}$-terms). The result is

$$
\begin{aligned}
& \bar{\Delta}_{D_{0}}\left[I_{2,2}\left(A_{1}, A_{2} ; D_{0}, D_{1}\right)\right]=-f_{1} \frac{\left(A_{1} \cdot q\right)\left(A_{2} \cdot q\right)}{\left(K_{1} \cdot q\right)^{2}} \Delta_{D_{0}}\left[\frac{1}{D_{0}}\right], \\
& \tilde{\Delta}_{D_{0}}\left[I_{2,2}\left(A_{1}, A_{2} ; D_{0}, D_{1}\right)\right]=-2 \pi K^{2} \Lambda^{4} \frac{\left(A_{1} \cdot q\right)\left(A_{2} \cdot q\right)}{\left(K_{1} \cdot q\right)} t^{2} .
\end{aligned}
$$

For later convenience we compute $\bar{\Delta}_{m^{2}} I_{2,2}$ in the case where $K_{1}^{2}=0$ and $m_{0}^{2}=m_{1}^{2}=m^{2}$,

$$
\begin{aligned}
\bar{\Delta}_{m^{2}}\left[I_{2,2}\left(A_{1}, A_{2} ; D_{0}, D_{1}\right)\right] & =\bar{\Delta}_{D_{0}}\left[I_{2,2}\left(A_{1}, A_{2} ; D_{0}, D_{1}\right)\right]+\bar{\Delta}_{D_{1}}\left[I_{2,2}\left(A_{1}, A_{2} ; D_{0}, D_{1}\right)\right] \\
& =-\left\{\left(2 K_{1} \cdot A_{1}\right) \bar{\Delta}_{D_{0}}\left[I_{2,1}\left(A_{2} ; D_{0}, D_{1}\right)\right]+\left(A_{1} \leftrightarrow A_{2}\right)\right\} .
\end{aligned}
$$

\section{Computation of $\bar{\Delta}_{\mathrm{D}_{0}}\left[\mathbf{I}_{3,1}\right]$}

The integrand $K^{2} t^{2} G_{3,1}\left(A_{1} ; D_{0}, D_{1}, D_{2} ; \alpha\right)$ does not contain $\Lambda^{2} t$-terms. Therefore $\bar{\Delta}_{D_{0}}\left[I_{3,1}\right]$ vanishes.

$$
\bar{\Delta}_{D_{0}}\left[I_{3,1}\left(A_{1} ; D_{0}, D_{1}, D_{2}\right)\right]=0 .
$$

This result confirms the absence of tadpole integrals in the reduction of $I_{3,1}$.

\section{Computation of $\bar{\Delta}_{\mathrm{D}_{0}}\left[\mathbf{I}_{3,2}\right]$}

The expression of $G_{3,2}\left(A_{1}, A_{2} ; D_{0}, D_{1}, D_{2} ; \alpha\right)$ is rather complicated and is not shown. As in the previous cases, $K^{2} t^{3} G_{3,2}$ has to be expanded in the $\Lambda \rightarrow \infty$ limit. We obtain $\bar{\Delta}_{D_{0}}\left[I_{3,2}\right]$ by integrating the terms of $\mathcal{O}\left(\Lambda^{2} t\right)$, finding

$$
\bar{\Delta}_{D_{0}}\left[I_{3,2}\left(A_{1}, A_{2} ; D_{0}, D_{1}, D_{2}\right)\right]=\frac{\left(A_{1} \cdot q\right)\left(A_{2} \cdot q\right)}{\left(K_{1} \cdot q\right)\left(K_{2} \cdot q\right)} \Delta_{D_{0}}\left[\frac{1}{D_{0}}\right] .
$$

Computation of $\bar{\Delta}_{\mathrm{D}_{0}}\left[\mathbf{I}_{3,3}\right]$

We obtain $\bar{\Delta}_{D_{0}}\left[I_{3,3}\right]$ by taking the $\Lambda \rightarrow \infty$ limit of $K^{2} t^{4} G_{3,3}$ and integrating the $\Lambda^{2} t$-terms, to get

$$
\bar{\Delta}_{D_{0}}\left[I_{3,3}\left(A_{1}, A_{2}, A_{3} ; D_{0}, D_{1}, D_{2}\right)\right]=\sum_{i=1}^{2} f_{i} \frac{\left(A_{1} \cdot q\right)\left(A_{2} \cdot q\right)\left(A_{3} \cdot q\right)}{\left(K_{1} \cdot q\right)\left(K_{2} \cdot q\right)\left(K_{i} \cdot q\right)} \Delta_{D_{0}}\left[\frac{1}{D_{0}}\right] .
$$

\section{Computation of tadpole coefficients}

In this section we show how the single cut allows the computation of tadpole coefficients in several examples of small integrands.

We will compute $a(0)$, the coefficient of the tadpole integral $A_{0}\left(m_{0}^{2}\right)$, by the single cut operator $\bar{\Delta}_{D_{0}}$ defined in (2.23). We look at one-loop integrands of the type

$$
\mathcal{I}_{n, p} \equiv \frac{\prod_{i=1}^{p}\left(2 k \cdot R_{i}\right)}{\prod_{j=0}^{n-1} D_{j}} .
$$


As described in the setup, when we cut the propagator $D_{0}$, we set $K_{0}=0$. Any appearance of the contraction $k^{2}$ in the numerator should then immediately be replaced by $m_{0}^{2}$, so that this form of the integrand is in fact general.

For now, we assume that the Gram determinant of $\mathcal{I}_{n, p}$ is nonvanishing and that the masses are non-degenerate. When this is not the case, further modifications are necessary, which we address in the following section.

We will describe the derivation of the tadpole coefficients for the integrands with $(n, p)=\{(2,1),(2,2),(3,2),(3,3)\}$. We have also verified the result for $(n, p)=(4,3)$, but as this calculation does not involve any notable new features, we do not present it here. We have used FeynCalc [57] to check our results against those from Passarino-Veltman reduction.

The idea underlying our procedure is to expand the integrand in such a way that spurious terms are easily recognized. The Ossola-Papadopoulos-Pittau (OPP) decomposition [10] is well suited for this purpose. We take all the coefficients of both spurious and physical terms as unknowns. We then drop the physical terms except for the tadpole. Single cuts of all remaining terms are evaluated using the general results of the previous section. The tensor $\prod_{i} R_{i}$ is expanded in a basis constructed from fixed vectors; a convenient choice includes external momenta and orthogonal vectors, as in the OPP classification. Thanks to this expansion, the single-cut equation becomes a system of separate equations, which are the coefficients of independent tensors. In the following examples, we illustrate the derivation of the system and the solution of the tadpole coefficient.

\section{Tadpole coefficient of $\mathcal{I}_{2,1}$}

The starting point is the OPP decomposition of $\mathcal{I}_{2,1}$ given by

$$
\mathcal{I}_{2,1}=\frac{a(0)}{D_{0}}+\tilde{b}_{11}(01) \frac{2 k \cdot \ell_{7}}{D_{0} D_{1}}+\tilde{b}_{21}(01) \frac{2 k \cdot \ell_{8}}{D_{0} D_{1}}+\tilde{b}_{0}(01) \frac{2 k \cdot n}{D_{0} D_{1}}+\cdots
$$

Terms whose single cut contains no $\Lambda^{2} t$ contribution are included in "..." . These terms will be systematically neglected throughout this section. The momenta $n, \ell_{7}$ and $\ell_{8}$ are defined [10] to satisfy the conditions

$$
K_{1} \cdot n=K_{1} \cdot \ell_{7}=K_{1} \cdot \ell_{8}=0, \quad n^{2}=\ell_{7} \cdot \ell_{8}=-K_{1}^{2}, \quad \ell_{7}^{2}=\ell_{8}^{2}=0 .
$$

Applying the single cut operator $\bar{\Delta}_{D_{0}}$ and using (3.7), we get

$$
\begin{aligned}
0 & =\left[-\left(a(0)+\alpha_{1}\right) K_{1}^{\mu}+\left(\tilde{b}_{11}(01)-\alpha_{3}\right) \ell_{7}^{\mu}\right. \\
& \left.+\left(\tilde{b}_{21}(01)-\alpha_{4}\right) \ell_{8}^{\mu}+\left(\tilde{b}_{0}(01)-\alpha_{2}\right) n^{\mu}\right] \frac{q_{\mu}}{K_{1} \cdot q} \Delta_{D_{0}}\left[\frac{1}{D_{0}}\right]
\end{aligned}
$$

Here $\alpha_{i=1, \cdots, 4}$ are the coordinates of $R_{1}$ in the basis $\left\{K_{1}, n, \ell_{7}, \ell_{8}\right\}$. Explicitly, they read as follows:

$$
\alpha_{1}=\frac{R_{1} \cdot K_{1}}{K_{1}^{2}}, \quad \alpha_{2}=-\frac{R_{1} \cdot n}{K_{1}^{2}}, \quad \alpha_{3}=-\frac{R_{1} \cdot \ell_{8}}{K_{1}^{2}}, \quad \alpha_{4}=-\frac{R_{1} \cdot \ell_{7}}{K_{1}^{2}} .
$$


From the relation (4.3) we see our first example of a system of equations leading to the tadpole coefficient. Since $q$ can be chosen arbitrarily, the expression inside the square brackets vanishes. This implies that each of the factors multiplying the basis vectors vanishes separately, giving four equations.

In fact, we only need the first of these equations to get the tadpole coefficient; here we do not need to solve for any of the spurious coefficients. The result is

$$
a(0)+\alpha_{1}=0 \Longrightarrow a(0)=-\frac{R_{1} \cdot K_{1}}{K_{1}^{2}}
$$

This value is in accordance with the one obtained using the Passarino-Veltman decomposition.

\section{Tadpole coefficient of $\mathcal{I}_{2,2}$}

For $\mathcal{I}_{2,2}$, again we use the OPP expansion, keeping only the terms with a non-vanishing single cut of $D_{0}$. These are the tadpole along with spurious terms,

$$
\begin{aligned}
\mathcal{I}_{2,2} & =\frac{a(0)}{D_{0}}+\tilde{b}_{11}(01) \frac{2 k \cdot \ell_{7}}{D_{0} D_{1}}+\tilde{b}_{21}(01) \frac{2 k \cdot \ell_{8}}{D_{0} D_{1}}+\tilde{b}_{0}(01) \frac{2 k \cdot n}{D_{0} D_{1}} \\
& +\tilde{b}_{12}(01) \frac{\left(2 k \cdot \ell_{7}\right)^{2}}{D_{0} D_{1}}+\tilde{b}_{22}(01) \frac{\left(2 k \cdot \ell_{8}\right)^{2}}{D_{0} D_{1}}+\tilde{b}_{01}(01) \frac{\left(2 k \cdot \ell_{7}\right)(2 k \cdot n)}{D_{0} D_{1}} \\
& +\tilde{b}_{02}(01) \frac{\left(2 k \cdot \ell_{8}\right)(2 k \cdot n)}{D_{0} D_{1}}+\tilde{b}_{00}(01)\left[\frac{(2 k \cdot n)^{2}}{D_{0} D_{1}}-\frac{\left(2 k \cdot K_{1}\right)^{2}-4 k^{2} K_{1}^{2}}{3 D_{0} D_{1}}\right]+\cdots .
\end{aligned}
$$

We compute the single cut of both sides of (4.6) using (3.7) and (3.10). The outcome is

$$
\begin{aligned}
0 & =\left[-\left(\frac{a(0)}{f_{1}}+\alpha_{1}+\frac{\tilde{b}_{00}(01)}{3}\right) K_{1}^{\mu} K_{1}^{\nu}+\left(\tilde{b}_{00}(01)-\alpha_{2}\right) n^{\mu} n^{\nu}+\left(\tilde{b}_{12}(01)-\alpha_{3}\right) \ell_{7}^{\mu} \ell_{7}^{\nu}\right. \\
& +\left(\tilde{b}_{22}(01)-\alpha_{4}\right) \ell_{8}^{\mu} \ell_{8}^{\nu}+\left(\frac{\tilde{b}_{0}(01)}{f_{1}}-2 \alpha_{5}\right) K_{1}^{\mu} n^{\nu}+\left(\frac{\tilde{b}_{11}(01)}{f_{1}}-2 \alpha_{7}\right) K_{1}^{\mu} \ell_{7}^{\nu} \\
& +\left(\frac{\tilde{b}_{21}(01)}{f_{1}}-2 \alpha_{8}\right) K_{1}^{\mu} \ell_{8}^{\nu}+\left(\tilde{b}_{01}(01)-2 \alpha_{13}\right) n^{\mu} \ell_{7}^{\nu} \\
& \left.+\left(\tilde{b}_{02}(01)-2 \alpha_{14}\right) n^{\mu} \ell_{8}^{\nu}\right] \Delta_{D_{0}}\left[\frac{1}{D_{0}}\right] \frac{q_{\mu} q_{\nu}}{\left(K_{1} \cdot q\right)^{2}} f_{1} .
\end{aligned}
$$

Here $f_{1}$ is defined according to equation (2.12), while $\alpha_{i=1, \ldots, 16}$ are obtained from the following decomposition of $R_{1}^{\mu} R_{2}^{\nu}$ in a basis of independent tensors:

$$
\begin{aligned}
R_{1}^{\mu} R_{2}^{\nu} & =\alpha_{1} K_{1}^{\mu} K_{1}^{\nu}+\alpha_{2} n^{\mu} n^{\nu}+\sum_{i=7}^{8}\left[\alpha_{i-4} \ell_{i}^{\mu} \ell_{i}^{\nu}\right]+\alpha_{5}\left(K_{1}^{\mu} n^{\nu}+K_{1}^{\nu} n^{\mu}\right)+\alpha_{6}\left(K_{1}^{\mu} n^{\nu}-K_{1}^{\nu} n^{\mu}\right) \\
& +\sum_{i=7}^{8}\left[\alpha_{i}\left(K_{1}^{\mu} \ell_{i}^{\nu}+K_{1}^{\nu} \ell_{i}^{\mu}\right)+\alpha_{i+2}\left(K_{1}^{\mu} \ell_{i}^{\nu}-K_{1}^{\nu} \ell_{i}^{\mu}\right)\right]+\alpha_{11}\left(\ell_{7}^{\mu} \ell_{8}^{\nu}-\ell_{7}^{\nu} \ell_{8}^{\mu}\right)+\alpha_{12} g_{\mu \nu} \\
& +\sum_{i=7}^{8}\left[\alpha_{i+6}\left(n^{\mu} \ell_{i}^{\nu}+n^{\nu} \ell_{i}^{\mu}\right)+\alpha_{i+8}\left(n^{\mu} \ell_{i}^{\nu}-n^{\nu} \ell_{i}^{\mu}\right)\right]
\end{aligned}
$$


The condition (4.7) is fullfilled for any lightlike $q$ only if the second rank tensor inside the square brackets vanishes. The first independent tensor includes the tadpole coefficient, but also the spurious coefficient $\tilde{b}_{00}(01)$. So we need the second independent tensor as well, but no others. In particular,

$$
\left\{\begin{array}{l}
\frac{a(0)}{f_{1}}+\alpha_{1}+\frac{\tilde{b}_{00}(01)}{3}=0 \\
\tilde{b}_{00}(01)-\alpha_{2}=0
\end{array} \quad \Longrightarrow \quad a(0)=-f_{1}\left(\alpha_{1}+\frac{\alpha_{2}}{3}\right)\right.
$$

Using the explicit expression of $\alpha_{1}$ and $\alpha_{2}$,

$$
\begin{aligned}
& \alpha_{1}=\frac{3\left(K_{1} \cdot R_{1}\right)\left(K_{1} \cdot R_{2}\right)}{2\left(K_{1}^{2}\right)^{2}}-\frac{\left(R_{1} \cdot R_{2}\right)}{2 K_{1}^{2}}-\frac{\left(n \cdot R_{1}\right)\left(n \cdot R_{2}\right)}{2\left(K_{1}^{2}\right)^{2}} \\
& \alpha_{2}=-\frac{\left(K_{1} \cdot R_{1}\right)\left(K_{1} \cdot R_{2}\right)}{2\left(K_{1}^{2}\right)^{2}}+\frac{\left(R_{1} \cdot R_{2}\right)}{2 K_{1}^{2}}+\frac{3\left(n \cdot R_{1}\right)\left(n \cdot R_{2}\right)}{2\left(K_{1}^{2}\right)^{2}}
\end{aligned}
$$

we get the value of the tadpole coefficient,

$$
a(0)=\frac{f_{1}}{3\left(K_{1}^{2}\right)^{2}}\left(K_{1}^{2}\left(R_{1} \cdot R_{2}\right)-4\left(K_{1} \cdot R_{1}\right)\left(K_{1} \cdot R_{2}\right)\right)
$$

\section{Tadpole coefficient of $\mathcal{I}_{3,2}$}

We now find it convenient to vary the OPP decomposition slightly. Our single-cut decomposition of $\mathcal{I}_{3,2}$ reads as follows,

$$
\begin{aligned}
\mathcal{I}_{3,2} & =\frac{a(0)}{D_{0}}+\sum_{i=1}^{2} \tilde{c}_{i 2}(012) \frac{\left(2 k \cdot \ell_{i+2}\right)^{2}}{D_{0} D_{1} D_{2}}+\sum_{j=1}^{2} \sum_{i=1}^{2} \tilde{b}_{i 1}(0 j) \frac{2 k \cdot \ell_{i+2}}{D_{0} D_{j}} \\
& +\sum_{i=1}^{2} \tilde{b}_{0}(0 i)\left(\frac{2 k \cdot K_{3-i}}{D_{0} D_{i}}-\frac{K_{1} \cdot K_{2}}{K_{i}^{2}} \frac{2 k \cdot K_{i}}{D_{0} D_{i}}\right)+\cdots
\end{aligned}
$$

As in OPP, $\ell_{3}$ and $\ell_{4}$ are lightlike momenta such that

$$
\ell_{j}^{2}=K_{i} \cdot \ell_{j}=0, \quad \forall i \in\{1,2\}, j \in\{3,4\}
$$

The evaluation of the single cut is performed using equations (3.7), (3.10) and (3.14). The result can be written as follows,

$$
\begin{aligned}
0 & =\left[\sum_{i=1}^{2}\left(\tilde{c}_{i 2}(012)-\gamma_{i+2 i+2}^{0}\right) \ell_{i+2}^{\mu} \ell_{i+2}^{\nu}-\left(\tilde{b}_{11}(01)+2 \gamma_{23}^{+}\right) K_{2}^{\mu} \ell_{3}^{\nu}-\left(\tilde{b}_{11}(02)+2 \gamma_{13}^{+}\right) K_{1}^{\mu} \ell_{3}^{\nu}\right. \\
& -\left(\tilde{b}_{21}(01)+2 \gamma_{24}^{+}\right) K_{2}^{\mu} \ell_{4}^{\nu}-\left(\tilde{b}_{21}(02)+2 \gamma_{14}^{+}\right) K_{1}^{\mu} \ell_{4}^{\nu}-\left(\tilde{b}_{0}(02)+\gamma_{11}^{0}\right) K_{1}^{\mu} K_{1}^{\nu} \\
& \left.-\left(\tilde{b}_{0}(01)+\gamma_{22}^{0}\right) K_{2}^{\mu} K_{2}^{\nu}+\left(a(0)-2 \gamma_{12}^{+}+\tilde{b}(01) \frac{K_{1} \cdot K_{2}}{K_{1}^{2}}+\tilde{b}(02) \frac{K_{1} \cdot K_{2}}{K_{2}^{2}}\right) K_{1}^{\mu} K_{2}^{\nu}\right] \\
& \times \Delta_{D_{0}}\left[\frac{1}{D_{0}}\right] \frac{q_{\mu} q_{\nu}}{\left(K_{1} \cdot q\right)\left(K_{2} \cdot q\right)} .
\end{aligned}
$$


The coefficients $\gamma_{i j}^{\dddot{j}}$ are obtained from the decomposition of $R_{1}^{\mu} R_{2}^{\nu}$ in a basis of independent tensors,

$$
\begin{aligned}
R_{1}^{\mu} R_{2}^{\nu} & =\gamma_{00} g_{\mu \nu}+\sum_{i=1}^{2}\left[\gamma_{i i}^{0} K_{i}^{\mu} K_{i}^{\nu}+\gamma_{i+2 i+2}^{0} \ell_{i+2}^{\mu} \ell_{i+2}^{\nu}\right]+\gamma_{12}^{+}\left(K_{1}^{\mu} K_{2}^{\nu}+K_{1}^{\nu} K_{2}^{\mu}\right) \\
& +\sum_{i=1}^{2} \sum_{j=3}^{4}\left[\gamma_{i j}^{+}\left(K_{i}^{\mu} \ell_{j}^{\nu}+K_{i}^{\nu} \ell_{j}^{\mu}\right)+\gamma_{i j}^{-}\left(K_{i}^{\mu} \ell_{j}^{\nu}-K_{i}^{\nu} \ell_{j}^{\mu}\right)\right] \\
& +\gamma_{34}^{-}\left(\ell_{3}^{\mu} \ell_{4}^{\nu}-\ell_{3}^{\nu} \ell_{4}^{\mu}\right)+\gamma_{12}^{-}\left(K_{1}^{\mu} K_{2}^{\nu}-K_{1}^{\nu} K_{2}^{\mu}\right) .
\end{aligned}
$$

Eq (4.13) implies that the following conditions have to be fulfilled,

$$
\left\{\begin{array}{l}
\tilde{b}_{0}(01)+\gamma_{22}^{0}=0 \\
a(0)-2 \gamma_{12}^{+}+\tilde{b}_{0}(01) \frac{K_{1} \cdot K_{2}}{K_{1}^{2}}+\tilde{b}_{0}(02) \frac{K_{1} \cdot K_{2}}{K_{2}^{2}}=0 \\
\tilde{b}_{0}(02)+\gamma_{11}^{0}=0
\end{array}\right.
$$

The system (4.15) together with the explicit expressions of $\gamma_{11}^{0}, \gamma_{22}^{0}$ and $\gamma_{12}^{+}$, gives the tadpole coefficient of $\mathcal{I}_{3,2}$,

$$
a(0)=2 \gamma_{12}^{+}+\gamma_{22}^{0} \frac{K_{1} \cdot K_{2}}{K_{1}^{2}}+\gamma_{11}^{0} \frac{K_{1} \cdot K_{2}}{K_{2}^{2}}=\frac{\sum_{i, j=1}^{2} b_{i j}\left(K_{i} \cdot R_{1}\right)\left(K_{j} \cdot R_{2}\right)}{K_{1}^{2} K_{2}^{2}\left(\left(K_{1} \cdot K_{2}\right)^{2}-K_{1}^{2} K_{2}^{2}\right)} .
$$

The factors $b_{i j}$ appearing in (4.16) are defined as follows,

$$
b_{12}=b_{21}=-K_{1}^{2} K_{2}^{2}, \quad b_{11}=K_{2}^{2}\left(K_{1} \cdot K_{2}\right), \quad b_{22}=K_{1}^{2}\left(K_{1} \cdot K_{2}\right) .
$$

The decomposition (4.11) relies on the particular structure of the numerator of $\mathcal{I}_{3,2}$. Being more general, the OPP decomposition does not take advantage of the knowledge of the numerator of $\mathcal{I}_{3,2}$. As a consequence, new spurious terms enter. They are of the type

$$
\frac{(2 k \cdot P)(2 k \cdot Q)}{D_{0} D_{i}}, \quad \frac{2 k \cdot P}{D_{0}}
$$

with $P, Q \in\left\{K_{1}, K_{2}, \ell_{3}, \ell_{4}\right\}$ and $i=1,2$. The coefficients of the terms (4.16) vanish. This can be explicitly shown using the operator $\tilde{\Delta}_{D_{0}}$ defined in (2.24), which selects the $\Lambda^{4} t^{4}$-enhanced terms of the single cut of $\mathcal{I}_{3,2}$.

\section{Tadpole coefficient of $\mathcal{I}_{3,3}$}

This is the last example of a tadpole coefficient that we will describe. We have done the analogous calculation for $\mathcal{I}_{4,3}$, but it does not introduce any notable new features.

Here again, although we could have started with the full OPP expansion or other variations, we can simplify the calculation by the particular expansion of $\mathcal{I}_{3,3}$ in the following 
tensor integrands:

$$
\begin{aligned}
\mathcal{I}_{3,3} & =\frac{a(0)}{D_{0}}+\sum_{i=3}^{4} \tilde{c}_{i i i} \frac{\left(2 k \cdot \ell_{i}\right)^{3}}{D_{0} D_{1} D_{2}}+\sum_{i=3}^{4} \sum_{j=1}^{2} \tilde{c}_{i i j} \frac{\left(2 k \cdot \ell_{i}\right)\left(2 k \cdot \ell_{i}\right)\left(2 k \cdot K_{j}\right)}{D_{0} D_{1} D_{2}} \\
& +\sum_{i=3}^{4} \sum_{j=1}^{2} \sum_{k=j}^{2} \tilde{c}_{i j k} \frac{\left(2 k \cdot \ell_{i}\right)\left(2 k \cdot K_{j}\right)\left(2 k \cdot K_{k}\right)}{D_{0} D_{1} D_{2}} \\
& -\sum_{i, j=1}^{2} \tilde{c}_{i i j}\left(f_{j}\left[\frac{\left(2 k \cdot K_{i}\right)}{D_{0} D_{3-i}}\right]_{\mathrm{sp} .}+\left[\frac{\left(2 k \cdot K_{i}\right)^{2}}{D_{0} D_{3-i}}\right]_{\mathrm{sp} .} \delta_{i j}\right)+\cdots .
\end{aligned}
$$

Here we are using the definition of $\ell_{3}$ and $\ell_{4}$ given in (4.12). The operator $[\cdots]_{\mathrm{sp}}$. selects the spurious parts of its argument. The explicit expressions of the spurious part can be read from equations (4.2) and (4.6). The single cut of both sides of equation (4.17) is computed using (3.7), (3.10), and (3.15). Using the decomposition of $R_{1}^{\mu} R_{2}^{\nu} R_{3}^{\sigma}$ in a basis of independent tensors,

$$
\begin{aligned}
R_{1}^{\mu} R_{2}^{\nu} R_{3}^{\sigma} & =\sum_{i, j, k=1}^{2} \alpha_{i j k} K_{i}^{\mu} K_{j}^{\nu} K_{k}^{\sigma}+\sum_{i=3}^{4} \alpha_{i i i} \ell_{i}^{\mu} \ell_{i}^{\nu} \ell_{i}^{\sigma}+\sum_{i=3}^{4}\left(\alpha_{i 00}^{+} \ell_{i}^{\mu} g^{\nu \sigma}+\alpha_{00 i}^{+} \ell_{i}^{\sigma} g^{\mu \nu}\right. \\
& \left.+\alpha_{i 00}^{-} \ell_{i}^{\mu}\left(\ell_{3}^{\nu} \ell_{4}^{\sigma}-\ell_{4}^{\nu} \ell_{3}^{\sigma}\right)\right)+\sum_{i=1}^{2}\left(\alpha_{i 00}^{+} K_{i}^{\mu} g^{\nu \sigma}+\alpha_{0 i 0}^{+} K_{i}^{\nu} g^{\mu \sigma}+\alpha_{00 i}^{+} K_{i}^{\sigma} g^{\mu \nu}\right) \\
& +\sum_{i=1}^{2}\left(\alpha_{i 00}^{-} K_{i}^{\mu}\left(\ell_{3}^{\nu} \ell_{4}^{\sigma}-\ell_{4}^{\nu} \ell_{3}^{\sigma}\right)+\alpha_{0 i 0}^{-} K_{i}^{\nu}\left(\ell_{3}^{\mu} \ell_{4}^{\sigma}-\ell_{4}^{\mu} \ell_{3}^{\sigma}\right)+\alpha_{00 i}^{-} K_{i}^{\sigma}\left(\ell_{3}^{\mu} \ell_{4}^{\nu}-\ell_{4}^{\mu} \ell_{3}^{\nu}\right)\right) \\
& +\sum_{i, j=1}^{2} \sum_{k=3}^{4}\left(\alpha_{i j k} K_{i}^{\mu} K_{j}^{\nu} \ell_{k}^{\sigma}+\alpha_{i k j} K_{i}^{\mu} K_{j}^{\sigma} \ell_{k}^{\nu}+\alpha_{k i j} K_{i}^{\nu} K_{j}^{\sigma} \ell_{k}^{\mu}\right) \\
& +\sum_{i=1}^{2} \sum_{j=3}^{4}\left(\alpha_{i j j} K_{i}^{\mu} \ell_{j}^{\nu} \ell_{j}^{\sigma}+\alpha_{j i j} K_{i}^{\nu} \ell_{j}^{\sigma} \ell_{j}^{\mu}+\alpha_{j j i} K_{i}^{\sigma} \ell_{j}^{\nu} \ell_{j}^{\mu}\right)
\end{aligned}
$$

and equating the single cuts on both sides of equation (4.17), we get the following relation:

$$
\begin{aligned}
0= & \sum_{m=1}^{2}\left\{\left[\sum_{i=3}^{4} \hat{c}_{i i i} \ell_{i}^{\mu} \ell_{i}^{\nu} \ell_{i}^{\sigma}+\sum_{i=3}^{4} \sum_{j=1}^{2} \sum_{k=j}^{2} \hat{c}_{i j k} \ell_{i}^{\mu} K_{j}^{\nu} K_{k}^{\sigma}+\sum_{i=3}^{4} \sum_{k=1}^{2} \hat{c}_{i i k} \ell_{i}^{\mu} \ell_{i}^{\nu} K_{k}^{\sigma}\right.\right. \\
+ & \sum_{i=1}^{2} g_{i i ; m}(\hat{c}) K_{i}^{\mu} K_{i}^{\nu} K_{i}^{\sigma}+g_{3-m m ; m}(\hat{c}) K_{3-m}^{\mu} K_{3-m}^{\nu} K_{m}^{\sigma} \\
+ & \frac{1}{2 f_{m}} K_{m}^{\mu} K_{m}^{\nu} K_{3-m}^{\sigma}\left(a(0)-\sum_{i, j, k=1}^{2} \alpha_{i j k} d_{i j k}\right. \\
& \left.\left.\left.+2 f_{m} g_{m} 3-m ; m(\hat{c})\right)\right] \Delta_{D_{0}}\left[\frac{1}{D_{0}}\right] \frac{q_{\mu} q_{\nu} q_{\sigma} f_{m}}{\left(K_{1} \cdot q\right)\left(K_{2} \cdot q\right)\left(K_{m} \cdot q\right)}\right\}
\end{aligned}
$$


The coefficients in the above relation have been defined for convenience of displaying the independent tensors. We use the following abbreviations $(i \neq j \neq k \neq i)$,

$$
\begin{aligned}
\hat{c}_{i i i} & =\tilde{c}_{i i i}-\alpha_{i i i} \\
\hat{c}_{i i k} & =\tilde{c}_{i i k}-\left(\alpha_{k i i}+\alpha_{i k i}+\alpha_{i i k}\right) \\
\hat{c}_{i j k} & =\tilde{c}_{i j k}-\left[\alpha_{k j i}+\alpha_{k i j}+\alpha_{i k j}+\left(1-\delta_{j k}\right)\left(\alpha_{j k i}+\alpha_{j i k}+\alpha_{i j k}\right)\right],
\end{aligned}
$$

and the following definition of the totally symmetric coefficient $d_{i j k}$,

$$
d_{i i i}=f_{i} \frac{K_{i} \cdot K_{3-i}}{K_{3-i}^{2}}-\frac{f_{3-i}}{3}\left(\frac{K_{i}^{2}}{K_{3-i}^{2}}-4 \frac{\left(K_{3-i} \cdot K_{i}\right)^{2}}{\left(K_{3-i}^{2}\right)^{2}}\right), \quad d_{i i j}=f_{i}+f_{j} \frac{K_{i} \cdot K_{j}}{K_{j}^{2}} .
$$

The $g_{i j ; m}(\hat{c})$ are given by

$$
\begin{aligned}
g_{i j ; m}(\hat{c}) & =\delta_{i j} \hat{c}_{i i i}+\left(1-\delta_{i j}\right)\left\{\delta_{m j} \hat{c}_{i i j}-\delta_{m i}\left[\frac{K_{1} \cdot K_{2}}{K_{j}^{2}} \hat{c}_{i i i}\right.\right. \\
& \left.\left.-\frac{1}{3\left(K_{i}^{2}\right)^{2}}\left(K_{1}^{2} K_{2}^{2}-4\left(K_{1} \cdot K_{2}\right)^{2}\right) \hat{c}_{j j j}+\frac{K_{1} \cdot K_{2}}{K_{i}^{2}} \hat{c}_{j j i}\right]\right\} .
\end{aligned}
$$

Since $q$ is arbitrary, we are led to the following relations.

$$
\left\{\begin{array}{l}
g_{11 ; 2}(\hat{c})=0 \\
g_{22 ; 1}(\hat{c})=0 \\
f_{1} g_{11 ; 1}(\hat{c})+f_{2} g_{12 ; 2}(\hat{c})=0 \\
f_{1} g_{21 ; 1}(\hat{c})+f_{2} g_{22 ; 2}(\hat{c})=0 \\
a(0)-\sum_{i, j, k=1}^{2} \alpha_{i j k} d_{i j k}+f_{1} g_{12 ; 1}(\hat{c})+f_{2} g_{21 ; 2}(\hat{c})=0
\end{array}\right.
$$

which uniquely fix $a(0)$,

$$
a(0)=\sum_{i, j, k=1}^{2} \alpha_{i j k} d_{i j k}
$$

We have observed that a different choice of the original integrand expansion, rather than (4.17), can lead to a larger linear system which is not completely solvable, yet there is still a unique solution for $a(0)$. The underlying phenomenon is that different spurious terms can have the same single cut, so these terms should really be grouped together in the expansion.

\section{Massless external legs}

As in other techniques such as Passarino-Veltman [21-25, 29, 30, 35, 58, 59] or OssolaPapadopoulos-Pittau $[10,50]$, we must modify our algorithm in the case of vanishing Gram determinants. The most immediate case is the presence of a massless external leg in tensor bubble integrals. In this section we will focus on this class of integrals in the case where the internal masses are the same, $m_{0}^{2}=m_{1}^{2}=m^{2}$. 
New features appear in the computation of $a_{\text {tot }}$, the coefficient of $A_{0}\left(m^{2}\right)$. First of all, if $K_{1}$ is light-like, it is not possible to complete a basis defining $n, \ell_{7}, \ell_{8}$. A suitable basis is given by $\left\{K_{1}, K_{2}, \ell_{7}, \ell_{8}\right\}$, which is composed of four light-like momenta such that

$$
K_{1} \cdot K_{2} \neq 0 \neq \ell_{7} \cdot \ell_{8}, \quad K_{i} \cdot \ell_{j}=0, \quad \forall i \in\{1,2\}, j \in\{7,8\} .
$$

Secondly, the coefficients of $1 / D_{0}$ and $1 / D_{1}$ contribute to $a_{\text {tot }}$ since

$$
\int d^{4} k \frac{1}{D_{0}}=\int d^{4} k \frac{1}{D_{1}}=A_{0}\left(m^{2}\right)
$$

Moreover the scalar bubble and the tadpole are connected,

$$
B_{0}\left(0, m^{2}, m^{2}\right)=\frac{A_{0}\left(m^{2}\right)}{m^{2}}-1,
$$

so the bubble coefficient contributes to the total tadpole coefficient. Tensor integrals contracted with $K_{2}$ are no longer spurious terms [50] and they contribute to the total tadpole coefficient. This can be easily understood looking at their explicit expression [35],

$$
\begin{aligned}
& \int d^{4} k \frac{\left(2 k \cdot K_{2}\right)}{D_{0} D_{1}}=B_{1}\left(0, m^{2}, m^{2}\right)\left(2 K_{2} \cdot K_{1}\right)=\frac{1}{2}\left(\frac{A_{0}\left(m^{2}\right)}{m^{2}}-1\right)\left(2 K_{2} \cdot K_{1}\right), \\
& \int d^{4} k \frac{\left(2 k \cdot K_{2}\right)^{2}}{D_{0} D_{1}}=B_{11}\left(0, m^{2}, m^{2}\right)\left(2 K_{2} \cdot K_{1}\right)^{2}=\frac{1}{3}\left(\frac{A_{0}\left(m^{2}\right)}{m^{2}}-1\right)\left(2 K_{2} \cdot K_{1}\right)^{2} .
\end{aligned}
$$

The tadpole coefficient $a_{\text {tot }}$ is obtained by summing the aforementioned contributions. These contributions can be obtained by applying the single cut operators defined in (2.23), (2.24), and (2.25).

\section{Tadpole coefficient of $\mathcal{I}_{2,1}$}

The full integrand is expanded as

$$
\mathcal{I}_{2,1}=\frac{a(0)}{D_{0}}+\frac{a(1)}{D_{1}}+\frac{b}{D_{0} D_{1}}+\hat{b}_{0}(01) \frac{\left(2 k \cdot K_{2}\right)}{D_{0} D_{1}}+\sum_{i=1}^{2} \tilde{b}_{i 1}(01) \frac{\left(2 k \cdot \ell_{i+6}\right)}{D_{0} D_{1}}+\cdots .
$$

The terms denoted by "..." are those such that $\bar{\Delta}_{m^{2}}[\cdots]=\bar{\Delta}_{D_{0}}[\cdots]=\hat{\Delta}_{D_{0}}[\cdots]=0$. The tadpole coefficient is given by

$$
a_{\mathrm{tot}}=a(0)+a(1)+\frac{b}{m^{2}}+\frac{\hat{b}_{0}(01)}{2 m^{2}}\left(2 K_{2} \cdot K_{1}\right) .
$$

The sum $a(0)+a(1)$ can be obtained by applying $\bar{\Delta}_{m^{2}}$. Using equation (3.9) we see that only the tadpole terms survive, so

$$
a(0)+a(1)=0 .
$$

The coefficient $\hat{b}_{0}(01)$ is obtained by cutting the propagator $D_{0}$ and selecting the $\Lambda^{2} t$ terms. The outcome is

$$
\begin{aligned}
0 & =\left[-\left(a(0)+\alpha_{1}\right) K_{1}^{\mu}+\left(\hat{b}_{0}(01)-\alpha_{2}\right) K_{2}^{\mu}\right. \\
& \left.+\sum_{i=1}^{2}\left(\tilde{b}_{i 1}(01)-\alpha_{i+2}\right) \ell_{i+6}^{\mu}\right] \frac{q^{\mu}}{K_{1} \cdot q} \Delta_{D_{0}}\left[\frac{1}{D_{0}}\right]
\end{aligned}
$$


The parameters $\alpha_{1, \cdots, 4}$ are the coordinates of $R_{1}$ in the basis $\left\{K_{1}, K_{2}, \ell_{7}, \ell_{8}\right\}$, which read as follows:

$$
\alpha_{1}=\frac{R_{1} \cdot K_{2}}{K_{1} \cdot K_{2}}, \quad \alpha_{2}=\frac{R_{1} \cdot K_{1}}{K_{1} \cdot K_{2}}, \quad \alpha_{3}=\frac{R_{1} \cdot \ell_{8}}{\ell_{7} \cdot \ell_{8}}, \quad \alpha_{4}=\frac{R_{1} \cdot \ell_{7}}{\ell_{7} \cdot \ell_{8}} .
$$

Since $q$ is arbitrary, each coefficient of the momenta appearing in (5.8) has to vanish. In particular,

$$
\hat{b}_{0}(01)=\alpha_{2}=\frac{R_{1} \cdot K_{1}}{K_{1} \cdot K_{2}}
$$

and $\tilde{b}_{i 1}(01)=\alpha_{i+2}$. Finally, the bubble coefficient $b$ can be obtained by using the operator $\hat{\Delta}_{D_{0}}$ defined in (2.24). Using equation (3.8), we get

$$
\begin{aligned}
0= & \sum_{a} \int d t c_{a}(t) v_{a \mu}\left[-\alpha_{1} K_{1}^{\mu}+\left(\hat{b}_{0}(01)-\alpha_{2}\right) K_{2}^{\mu}+\sum_{i=1}^{2}\left(\tilde{b}_{i 1}(01)-\alpha_{i+2}\right) \ell_{i+6}^{\mu}\right] \\
& +b \Delta_{D_{0}}\left[\frac{1}{D_{0} D_{1}}\right] \\
= & -\alpha_{1} \sum_{a} \int d t c_{a}(t)\left(v_{a} \cdot K_{1}\right)+b \Delta_{D_{0}}\left[\frac{1}{D_{0} D_{1}}\right] \\
= & b \Delta_{D_{0}}\left[\frac{1}{D_{0} D_{1}}\right] \Longrightarrow b=0 .
\end{aligned}
$$

The tadpole coefficient is obtained from equation (5.6), using the results (5.7), (5.10), and (5.11), and it is given by

as expected from explicit reduction.

$$
a_{\mathrm{tot}}=\frac{\left(R_{1} \cdot K_{1}\right)}{m^{2}},
$$

\section{Tadpole coefficient of $\mathcal{I}_{2,2}$}

Our final example is given to display the flexibility of single cut operations and the degrees of information available from cutting the same propagator. We use several different refinements of the single cut, picking out terms with different dependence on $\Lambda$ and $t$, in order to collect the subset of the information required for the tadpole coefficient.

The integrand $\mathcal{I}_{2,2}$ is decomposed as follows,

$$
\begin{aligned}
\mathcal{I}_{2,2} & =\frac{a(0)}{D_{0}}+\frac{a(1)}{D_{1}}+\frac{b}{D_{0} D_{1}}+\sum_{i=1}^{2}\left(\tilde{a}_{i}(0) \frac{2 k \cdot K_{i}}{D_{0}}+\tilde{a}_{i+2}(0) \frac{2 k \cdot \ell_{i+6}}{D_{0}}\right) \\
& +\sum_{i=1}^{2}\left(\tilde{b}_{0 i}(01) \frac{\left(2 k \cdot K_{2}\right)\left(2 k \cdot \ell_{i+6}\right)}{D_{0} D_{1}}+\tilde{b}_{i 2}(01) \frac{\left(2 k \cdot \ell_{i+6}\right)^{2}}{D_{0} D_{1}}\right) \\
& +\hat{b}_{00}(01) \frac{\left(2 k \cdot K_{2}\right)^{2}}{D_{0} D_{1}}+\cdots .
\end{aligned}
$$

The terms denoted by “..." are not explicitly shown since $\bar{\Delta}_{m^{2}}[\cdots]=\tilde{\Delta}_{D_{0}}[\cdots]=\hat{\Delta}_{D_{0}}[\cdots]=0$. The tadpole coefficient of $A_{0}\left(\mathrm{~m}^{2}\right)$ is given by

$$
a_{\mathrm{tot}}=a(0)+a(1)+\frac{b}{m^{2}}+\frac{\hat{b}_{00}(01)}{3 m^{2}}\left(2 K_{2} \cdot K_{1}\right)^{2} .
$$


In the computation we will take advantage of the following decomposition of $R_{1}^{\mu} R_{2}^{\nu}$ in independent tensors,

$$
\begin{aligned}
R_{1}^{\mu} R_{2}^{\nu} & =\sum_{i=1}^{2} \alpha_{i} K_{i}^{\mu} K_{i}^{\nu}+\sum_{i=7}^{8}\left[\alpha_{i-4} \ell_{i}^{\mu} \ell_{i}^{\nu}\right]+\alpha_{5}\left(K_{1}^{\mu} K_{2}^{\nu}+K_{1}^{\nu} K_{2}^{\mu}\right)+\alpha_{6}\left(K_{1}^{\mu} K_{2}^{\nu}-K_{1}^{\nu} K_{2}^{\mu}\right) \\
& +\sum_{i=7}^{8}\left[\alpha_{i}\left(K_{1}^{\mu} \ell_{i}^{\nu}+K_{1}^{\nu} \ell_{i}^{\mu}\right)+\alpha_{i+2}\left(K_{1}^{\mu} \ell_{i}^{\nu}-K_{1}^{\nu} \ell_{i}^{\mu}\right)\right]+\alpha_{11}\left(\ell_{7}^{\mu} \ell_{8}^{\nu}-\ell_{7}^{\nu} \ell_{8}^{\mu}\right) \\
& +\alpha_{12} g_{\mu \nu}+\sum_{i=7}^{8}\left[\alpha_{i+6}\left(K_{2}^{\mu} \ell_{i}^{\nu}+K_{2}^{\nu} \ell_{i}^{\mu}\right)+\alpha_{i+8}\left(K_{2}^{\mu} \ell_{i}^{\nu}-K_{2}^{\nu} \ell_{i}^{\mu}\right)\right]
\end{aligned}
$$

We apply the single cut and we select the $\Lambda^{2} t$ terms using the operator $\bar{\Delta}_{m^{2}}$ and equation (3.12).

$$
\begin{aligned}
0 & =\left[\left(\frac{a(0)+a(1)-4 \alpha_{12}}{2 K_{1} \cdot K_{2}}-2 \alpha_{5}\right) K_{1}^{\mu}+2\left(\hat{b}_{00}(01)-\alpha_{2}\right) K_{2}^{\mu}\right. \\
& \left.+\sum_{i=1}^{2}\left(\tilde{b}_{0 i}(01)-2 \alpha_{i+12}\right) \ell_{i+6}^{\mu}\right] \frac{2\left(K_{1} \cdot K_{2}\right) q_{\nu}}{K_{1} \cdot q} \Delta_{D_{0}}\left[\frac{1}{D_{0}}\right]
\end{aligned}
$$

Equation (5.16) fixes the values of $a(0)+a(1)$ and of $\hat{b}_{00}(01)$ to be

$$
\begin{aligned}
a(0)+a(1) & =4 \alpha_{12}+2 \alpha_{5}\left(2 K_{1} \cdot K_{2}\right), \\
\hat{b}_{00}(01) & =\alpha_{2} .
\end{aligned}
$$

The value of $\tilde{b}_{0 i}(01)$ is fixed as well, to be

$$
\tilde{b}_{0 i}(01)=2 \alpha_{i+12} .
$$

The value of $\tilde{b}_{i 2}(01)$ is read off from the $\Lambda^{4} t^{4}$-enhanced terms of the single cut of $\mathcal{I}_{2,2}$. These terms are selected using the operator $\tilde{\Delta}_{D_{0}}$ and using equations (3.6) and (3.11). The outcome can be written as follows,

$$
\begin{aligned}
0 & =\left[-\left(\tilde{a}_{1}(0)+\alpha_{1}\right) K_{1}^{\mu} K_{1}^{\mu}-\left(\tilde{a}_{2}(0)+2 \alpha_{5}\right) K_{1}^{\mu} K_{2}^{\mu}-\sum_{i=3}^{4}\left(\tilde{a}_{i}(0)+2 \alpha_{i+4}\right) K_{1}^{\mu} \ell_{i+4}^{\nu}\right. \\
& +\sum_{i=1}^{2}\left(\tilde{b}_{0 i}(01)-2 \alpha_{i+12}\right) K_{2}^{\mu} \ell_{i+6}^{\nu}+\sum_{i=1}^{2}\left(\tilde{b}_{i 2}(01)-\alpha_{i+2}\right) \ell_{i+6}^{\mu} \ell_{i+6}^{\nu} \\
& \left.+\left(\hat{b}_{00}(01)-\alpha_{2}\right) K_{2}^{\mu} K_{2}^{\mu}\right] \frac{2 q_{\mu} q_{\nu}}{K_{1} \cdot q} \pi K^{2} \Lambda^{4} t^{2} \\
& \Longrightarrow \tilde{b}_{i 2}(01)=\alpha_{i+2} .
\end{aligned}
$$

The logarithmically enhanced part of the single cut allows the computation of the bubble coefficient $b$. This is achieved by applying the $\hat{\Delta}_{D_{0}}$ operator defined in (2.24). Using equations (5.19) and (5.20), we find

$$
0=b \Delta_{D_{0}}\left[\frac{1}{D_{0} D_{1}}\right]-4 \alpha_{12} m^{2} \Delta_{D_{0}}\left[\frac{1}{D_{0} D_{1}}\right] \quad \Longrightarrow \quad b=4 m^{2} \alpha_{12} .
$$


Here we have used the property $\hat{\Delta}_{D_{0}} I_{2,2}\left(K_{1}, A_{2} ; D_{0}, D_{1}\right)=0$, which holds in the case $f_{1}=$ 0. The tadpole coefficient in Equation (5.14) is then obtained using equations (5.17), (5.18) and (5.21),

$$
a_{\text {tot }}=2\left(4 \alpha_{12}+\alpha_{5}\left(2 K_{1} \cdot K_{2}\right)\right)+\alpha_{2} \frac{\left(2 K_{2} \cdot K_{1}\right)^{2}}{3 m^{2}}=2 R_{1} \cdot R_{2}+\frac{\left(2 R_{1} \cdot K_{1}\right)\left(2 R_{2} \cdot K_{1}\right)}{3 m^{2}} .
$$

In obtaining the result (5.22), we used some explicit values of the coefficients in the expansion (5.15),

$$
4 \alpha_{12}+\alpha_{5}\left(2 K_{1} \cdot K_{2}\right)=\left(R_{1} \cdot R_{2}\right), \quad \alpha_{2}=\frac{\left(R_{1} \cdot K_{1}\right)\left(R_{2} \cdot K_{1}\right)}{\left(K_{1} \cdot K_{2}\right)^{2}} .
$$

\section{Conclusions and discussion}

We have seen how single cut integrals can distinguish scalar boxes, triangles, bubbles and especially tadpoles. We have outlined a strategy to find the tadpole coefficients applying single cuts. In particular we have used the fact that the null vector $q$ was chosen arbitrarily in order to establish the independence of contractions with it and derive a system of linear equations. The tadpole coefficient may been obtained as a solution of this system.

The general application of our procedure requires further investigation. Since single cuts of spurious terms do not vanish, we need to understand the single cuts of general integrands in order to isolate tadpole coefficients. We have made a start in this direction in section 3. It would be very interesting to generalize this analysis. The simple expressions of the terms proportional to $\Lambda^{2} t$ lead us to speculate that further results will be similarly simple. Perhaps there is even a more direct way to derive these terms in particular.

Although in the examples in section 4 , the system of equations derived from single cuts was sufficient to uniquely determine the tadpole coefficient, we need more general information about the system to be sure that it will always work. The rank of the matrix associated to the system of linear equation could be insufficient to find a unique solution for the tadpole coefficient. A possible concern is that $q$ is null and thus has fewer degrees of freedom than a generic vector. Here again, study of single cuts of general integrands could illuminate the role played by $q$ and perhaps resolve our concern about the general solvability of the linear system.

In Passarino-Veltman reduction, a single system of equations is solved for all coefficients of master integrals, and no spurious terms are involved. It is also unnecessary to reduce all the way to the tadpole level. In our procedure, by contrast, we actually solve two systems of equations: the first one is the expansion of the tensor numerator in a suitable basis of independent tensors. However, the second system leading to the tadpole coefficient is then quite simple and usually reducible. We have assumed that the coefficients of other master integrals would be obtained by (generalized) unitarity methods, so the total calculation is in fact quite long. We cannot claim that our method for getting tadpole coefficients will be efficient. Nevertheless, we have found it illuminating to investigate the single cut integral formalism in general. It would be very interesting to further probe the analytic structure of amplitudes, for example by developing a $D$-dimensional extension for studying their rational parts along the lines of [17]. 


\section{Acknowledgments}

We would like to thank P. Mastrolia and T. Robens for discussions inspiring this investigation. R.B. is supported by the Agence Nationale de la Recherche under grant ANR09-CEXC-009-01. E.M. is supported by the European Research Council under Advanced Investigator Grant ERC-AdG-228301.

\section{A Phase space parametrization}

In this appendix we describe some details of the evaluation of the single cut. The starting point is the integral

$$
\int_{\mathbb{R}^{4}} d^{4} \ell_{1} \delta^{+}\left(\ell_{1}^{2}\right) g
$$

where $g$ is a general integrand. Given an arbitrary four-vector $K$, such that $K^{2} \neq 0$ and its energy component is positive, $K_{0}>0$, we construct a pair of light-like momenta $p$ and $q$ such that $p+q=K$, and two more momenta $\epsilon_{1}, \epsilon_{2}$ defined as

$$
\epsilon_{1}=\frac{1}{2}\left(\left\langle q\left|\gamma^{\mu}\right| p\right]+\left\langle p\left|\gamma^{\mu}\right| q\right]\right), \quad \epsilon_{2}=\frac{1}{2 i}\left(\left\langle q\left|\gamma^{\mu}\right| p\right]-\left\langle p\left|\gamma^{\mu}\right| q\right]\right) .
$$

The loop momentum $\ell_{1}$ can be decomposed in the basis $\left\{p, q, \epsilon_{1}, \epsilon_{2}\right\}$,

$$
\ell_{1}=t\left(p+\alpha q+\epsilon_{1} x-\epsilon_{2} y\right),
$$

and the integral (A.1) can be expressed in terms of the coordinates $(t, \alpha, x, y)$. The Jacobian of this reparametrization reads as follows:

$$
\mathcal{J}=\left|-t^{3} \varepsilon_{\mu \nu \rho \sigma} p^{\mu} q^{\nu} \epsilon_{1}^{\rho} \epsilon_{2}^{\sigma}\right|=\frac{2\left(K^{2}\right)^{2}}{4}\left|t^{3}\right|,
$$

where in the last step the relation

$$
4 i \varepsilon^{\mu \nu \rho \sigma} a_{\mu} b_{\nu} c_{\rho} d_{\sigma}=-2\langle a|b c d| a]+(2 a \cdot b)(2 c \cdot d)-(2 a \cdot c)(2 b \cdot d)+(2 a \cdot d)(2 b \cdot c),
$$

has been used [60]. The integral (A.1) becomes

$$
\begin{aligned}
\int d^{4} \ell_{1} \delta^{+}\left(\ell_{1}^{2}\right) g & =\int d t d x d y d \alpha \mathcal{J} \delta^{+}\left(t^{2}\left(\alpha-x^{2}-y^{2}\right)\right) g \\
& =\int_{0}^{\infty} d t \int_{\mathbb{R}^{2}} d x d y \frac{K^{2} t}{2} g .
\end{aligned}
$$

This integral is computed using the generalized Cauchy formula,

$$
2 \pi i \sum_{\text {poles } z_{j}} \operatorname{Res}\left\{F(z, \bar{z}), z_{j}\right\}=\oint_{\partial S} F(z, \bar{z}) d z-\int_{S} \frac{\partial F}{\partial \bar{z}} d \bar{z} \wedge d z .
$$

Considering $g$ as a function of $(z, \bar{z})=(x+i y, x-i y)$, we construct its primitive $G(z, \bar{z})$ with respect to $\bar{z}$. Choosing $S$ to be the complex plane $\mathbb{C}$, we get

$$
\int_{\mathbb{R}^{2}} d x d y g=\frac{1}{2 i} \int_{\mathbb{C}} \frac{\partial G}{\partial \bar{z}} d \bar{z} \wedge d z=\frac{1}{2 i} \int_{\partial \mathbb{C}} G(z, \bar{z}) d z-\pi \sum_{\text {poles } z_{j}} \operatorname{Res}\left\{G(z, \bar{z}), z_{j}\right\} .
$$


In practice we regularize the divergences of the integral (A.4) taking instead of $\mathbb{C}$ a disk $D$ in the complex plane enclosing all poles of $G(z, \bar{z})$ viewed as a function of $z$. In polar coordinates the disk is parametrized as follows,

$$
z=r e^{i \alpha} ; \quad D=\{(r, \alpha) \mid 0 \leq r \leq \Lambda, 0 \leq \alpha<2 \pi\} .
$$

Under these assumptions, equation (A.4) becomes

$$
\int_{\mathbb{R}^{2}} d x d y g=\lim _{\Lambda \rightarrow \infty} \frac{1}{2} \int_{0}^{2 \pi} \Lambda e^{i \alpha} G\left(\Lambda e^{i \alpha}, \Lambda e^{-i \alpha}\right) d \alpha-\pi \sum_{\text {poles } z_{j}} \operatorname{Res}\left\{G(z, \bar{z}), z_{j}\right\} .
$$

In equations (A.2) and (A.4) the correct prefactors are kept, even though in unitarity methods these constant factors cancel out and can be neglected. ${ }^{2}$

Open Access. This article is distributed under the terms of the Creative Commons Attribution Noncommercial License which permits any noncommercial use, distribution, and reproduction in any medium, provided the original author(s) and source are credited.

\section{References}

[1] Z. Bern, L.J. Dixon, D.C. Dunbar and D.A. Kosower, One-loop n-point gauge theory amplitudes, unitarity and collinear limits, Nucl. Phys. B 425 (1994) 217 [hep-ph/9403226] [SPIRES].

[2] Z. Bern, L.J. Dixon, D.C. Dunbar and D.A. Kosower, Fusing gauge theory tree amplitudes into loop amplitudes, Nucl. Phys. B 435 (1995) 59 [hep-ph/9409265] [SPIRES].

[3] W.L. van Neerven, Dimensional regularization of mass and infrared singularities in two loop on-shell vertex functions, Nucl. Phys. B 268 (1986) 453 [SPIRES].

[4] R.E. Cutkosky, Singularities and discontinuities of Feynman amplitudes, J. Math. Phys. 1 (1960) 429 [SPIRES].

[5] Z. Bern, L.J. Dixon and D.A. Kosower, One-loop amplitudes for $e^{+} e^{-}$to four partons, Nucl. Phys. B 513 (1998) 3 [hep-ph/9708239] [SPIRES].

[6] Z. Bern, V. Del Duca, L.J. Dixon and D.A. Kosower, All non-maximally-helicity-violating one-loop seven-gluon amplitudes in $N=4$ super-Yang-Mills theory, Phys. Rev. D 71 (2005) 045006 [hep-th/0410224] [SPIRES].

[7] R. Britto, F. Cachazo and B. Feng, Generalized unitarity and one-loop amplitudes in $N=4$ super-Yang-Mills, Nucl. Phys. B 725 (2005) 275 [hep-th/0412103] [SPIRES].

[8] P. Mastrolia, On triple-cut of scattering amplitudes, Phys. Lett. B 644 (2007) 272 [hep-th/0611091] [SPIRES].

[9] D. Forde, Direct extraction of one-loop integral coefficients, Phys. Rev. D 75 (2007) 125019 [arXiv: 0704.1835] [SPIRES].

[10] G. Ossola, C.G. Papadopoulos and R. Pittau, Reducing full one-loop amplitudes to scalar integrals at the integrand level, Nucl. Phys. B 763 (2007) 147 [hep-ph/0609007] [SPIRES].

[11] R.K. Ellis, W.T. Giele and Z. Kunszt, A numerical unitarity formalism for evaluating one-loop amplitudes, JHEP 03 (2008) 003 [arXiv:0708.2398] [SPIRES].

\footnotetext{
${ }^{2}$ For instance, in the definition of the double cut of ref. [48] and related papers, a factor of $i / 4$ has been dropped. We thank T. Robens for pointing it out.
} 
[12] W.B. Kilgore, One-loop integral coefficients from generalized unitarity, arXiv:0711.5015 [SPIRES].

[13] W.T. Giele, Z. Kunszt and K. Melnikov, Full one-loop amplitudes from tree amplitudes, JHEP 04 (2008) 049 [arXiv:0801.2237] [SPIRES].

[14] C.F. Berger et al., An automated implementation of on-shell methods for one-loop amplitudes, Phys. Rev. D 78 (2008) 036003 [arXiv: 0803.4180] [SPIRES].

[15] S.D. Badger, Direct extraction of one loop rational terms, JHEP 01 (2009) 049 [arXiv:0806.4600] [SPIRES].

[16] R.K. Ellis, W.T. Giele, Z. Kunszt and K. Melnikov, Masses, fermions and generalized D-dimensional unitarity, Nucl. Phys. B 822 (2009) 270 [arXiv:0806.3467] [SPIRES].

[17] E.W. Nigel Glover and C. Williams, One-loop gluonic amplitudes from single unitarity cuts, JHEP 12 (2008) 067 [arXiv:0810.2964] [SPIRES].

[18] S. Caron-Huot, Loops and trees, arXiv:1007.3224 [SPIRES].

[19] S. Catani, T. Gleisberg, F. Krauss, G. Rodrigo and J.-C. Winter, From loops to trees by-passing Feynman's theorem, JHEP 09 (2008) 065 [arXiv:0804.3170] [SPIRES].

[20] I. Bierenbaum, S. Catani, P. Draggiotis and G. Rodrigo, A tree-loop duality relation at two loops and beyond, arXiv:1007.0194 [SPIRES].

[21] L.M. Brown and R.P. Feynman, Radiative corrections to Compton scattering, Phys. Rev. 85 (1952) 231 [SPIRES].

[22] D.B. Melrose, Reduction of Feynman diagrams, Nuovo Cim. 40 (1965) 181 [SPIRES].

[23] G. Passarino and M.J.G. Veltman, One loop corrections for $e^{+} e^{-}$annihilation into $\mu^{+} \mu^{-}$in the Weinberg model, Nucl. Phys. B 160 (1979) 151 [SPIRES].

[24] G. 't Hooft and M.J.G. Veltman, Scalar one loop integrals, Nucl. Phys. B 153 (1979) 365 [SPIRES].

[25] W.L. van Neerven and J.A.M. Vermaseren, Large loop integrals, Phys. Lett. B 137 (1984) 241 [SPIRES].

[26] R.G. Stuart, Algebraic reduction of one loop Feynman diagrams to scalar integrals, Comput. Phys. Commun. 48 (1988) 367 [SPIRES].

[27] R.G. Stuart and A. Gongora, Algebraic reduction of one loop Feynman diagrams to scalar integrals. 2, Comput. Phys. Commun. 56 (1990) 337 [SPIRES].

[28] G.J. van Oldenborgh and J.A.M. Vermaseren, New algorithms for one loop integrals, Z. Phys. C 46 (1990) 425 [SPIRES].

[29] Z. Bern, L.J. Dixon and D.A. Kosower, Dimensionally regulated one-loop integrals, Phys. Lett. B 302 (1993) 299 [hep-ph/9212308] [SPIRES].

[30] Z. Bern, L.J. Dixon and D.A. Kosower, Dimensionally regulated pentagon integrals, Nucl. Phys. B 412 (1994) 751 [hep-ph/9306240] [SPIRES].

[31] J. Fleischer, F. Jegerlehner and O.V. Tarasov, Algebraic reduction of one-loop Feynman graph amplitudes, Nucl. Phys. B 566 (2000) 423 [hep-ph/9907327] [SPIRES].

[32] T. Binoth, J.P. Guillet and G. Heinrich, Reduction formalism for dimensionally regulated one-loop N-point integrals, Nucl. Phys. B 572 (2000) 361 [hep-ph/9911342] [SPIRES]. 
[33] A. Denner and S. Dittmaier, Reduction of one-loop tensor 5-point integrals, Nucl. Phys. B 658 (2003) 175 [hep-ph/0212259] [SPIRES].

[34] G. Duplancic and B. Nizic, Reduction method for dimensionally regulated one-loop N-point Feynman integrals, Eur. Phys. J. C 35 (2004) 105 [hep-ph/0303184] [SPIRES].

[35] A. Denner and S. Dittmaier, Reduction schemes for one-loop tensor integrals, Nucl. Phys. B 734 (2006) 62 [hep-ph/0509141] [SPIRES].

[36] R.K. Ellis and G. Zanderighi, Scalar one-loop integrals for QCD, JHEP 02 (2008) 002 [arXiv:0712.1851] [SPIRES].

[37] Z. Bern and A.G. Morgan, Massive loop amplitudes from unitarity, Nucl. Phys. B 467 (1996) 479 [hep-ph/9511336] [SPIRES].

[38] S.D. Badger, Generalised unitarity at one-loop with massive fermions, Nucl. Phys. Proc. Suppl. 183 (2008) 220 [arXiv:0807.1245] [SPIRES].

[39] R. Britto and B. Feng, Solving for tadpole coefficients in one-loop amplitudes, Phys. Lett. B 681 (2009) 376 [arXiv:0904.2766] [SPIRES].

[40] R. Britto, E. Buchbinder, F. Cachazo and B. Feng, One-loop amplitudes of gluons in SQCD, Phys. Rev. D 72 (2005) 065012 [hep-ph/0503132] [SPIRES].

[41] R. Britto, B. Feng and P. Mastrolia, The cut-constructible part of QCD amplitudes, Phys. Rev. D 73 (2006) 105004 [hep-ph/0602178] [SPIRES].

[42] C. Anastasiou, R. Britto, B. Feng, Z. Kunszt and P. Mastrolia, D-dimensional unitarity cut method, Phys. Lett. B 645 (2007) 213 [hep-ph/0609191] [SPIRES].

[43] R. Britto and B. Feng, Unitarity cuts with massive propagators and algebraic expressions for coefficients, Phys. Rev. D 75 (2007) 105006 [hep-ph/0612089] [SPIRES].

[44] C. Anastasiou, R. Britto, B. Feng, Z. Kunszt and P. Mastrolia, Unitarity cuts and reduction to master integrals in d dimensions for one-loop amplitudes, JHEP 03 (2007) 111 [hep-ph/0612277] [SPIRES].

[45] R. Britto and B. Feng, Integral coefficients for one-loop amplitudes, JHEP 02 (2008) 095 [arXiv:0711.4284] [SPIRES].

[46] R. Britto, B. Feng and P. Mastrolia, Closed-form decomposition of one-loop massive amplitudes, Phys. Rev. D 78 (2008) 025031 [arXiv:0803.1989] [SPIRES].

[47] B. Feng and G. Yang, Unitarity method with spurious pole, Nucl. Phys. B 811 (2009) 305 [arXiv:0806.4016] [SPIRES].

[48] P. Mastrolia, Double-cut of scattering amplitudes and Stokes' theorem, Phys. Lett. B 678 (2009) 246 [arXiv:0905.2909] [SPIRES].

[49] N. Arkani-Hamed, J.L. Bourjaily, F. Cachazo, S. Caron-Huot and J. Trnka, The all-loop integrand for scattering amplitudes in planar $N=4$ SYM, arXiv:1008.2958 [SPIRES].

[50] G. Ossola, C.G. Papadopoulos and R. Pittau, Numerical evaluation of six-photon amplitudes, JHEP 07 (2007) 085 [arXiv: 0704.1271] [SPIRES].

[51] F. Cachazo, P. Svrček and E. Witten, MHV vertices and tree amplitudes in gauge theory, JHEP 09 (2004) 006 [hep-th/0403047] [SPIRES].

[52] F.A. Berends, R. Kleiss, P. De Causmaecker, R. Gastmans and T.T. Wu, Single Bremsstrahlung processes in gauge theories, Phys. Lett. B 103 (1981) 124 [SPIRES]. 
[53] P. De Causmaecker, R. Gastmans, W. Troost and T.T. Wu, Multiple Bremsstrahlung in gauge theories at high-energies. 1. General formalism for quantum electrodynamics, Nucl. Phys. B 206 (1982) 53 [SPIRES].

[54] R. Kleiss and W.J. Stirling, Spinor techniques for calculating $p \bar{p} \rightarrow W^{ \pm} / Z^{0}+$ Jets, Nucl. Phys. B 262 (1985) 235 [SPIRES].

[55] Z. Xu, D.-H. Zhang and L. Chang, Helicity amplitudes for multiple Bremsstrahlung in massless nonabelian gauge theories, Nucl. Phys. B 291 (1987) 392 [SPIRES].

[56] J.F. Gunion and Z. Kunszt, Improved analytic techniques for tree graph calculations and the G g q anti-q lepton anti-lepton subprocess, Phys. Lett. B 161 (1985) 333 [SPIRES].

[57] R. Mertig, M. Böhm and A. Denner, FEYN CALC: Computer algebraic calculation of Feynman amplitudes, Comput. Phys. Commun. 64 (1991) 345 [SPIRES].

[58] A. Denner, S. Dittmaier, M. Roth and L.H. Wieders, Complete electroweak $\mathcal{O}(\alpha)$ corrections to charged-current $e^{+} e^{-} \rightarrow 4$ fermion processes, Phys. Lett. B 612 (2005) 223 [hep-ph/0502063] [SPIRES].

[59] A. Denner, S. Dittmaier, M. Roth and L.H. Wieders, Electroweak corrections to charged-current $e^{+} e^{-} \rightarrow 4$ fermion processes: Technical details and further results, Nucl. Phys. B 724 (2005) 247 [hep-ph/0505042] [SPIRES].

[60] L.J. Dixon, Calculating scattering amplitudes efficiently, hep-ph/9601359 [SPIRES]. 